\title{
THINKING DEVELOPMENT AND CULTURAL AWARENESS OF STUDENTS THROUGH LITERATURE
}

\section{DESARROLLAR EL PENSAMIENTO Y AUMENTAR LA CONCIENCIA CULTURAL DE LOS ESTUDIANTES A TRAVÉS DE LA LITERATURA}

\author{
Adriana Lăzărescu*, ORCID ID: 0000-0001-7547-0793 \\ University of Craiova, 13 Al Cuza Str., Craiova, Romania \\ *Corresponding author: Adriana Lăzărescu, adriana_3003@yahoo.com
}

Received: 06. 12. 2021

Accepted: 07. 25. 2021

\begin{abstract}
The purpose of present research is to emphasize the way in which literature contributes to the needs of new students in their social interaction. The key factors that we focused on span the entire scope of studying a foreign language through literature. Thus, the methods we suggest for this refer to: motivating students to read, using poems, creating a play in class, using dramatic texts and narratives. It has been constantly recognized that the new European context influences the new generation of students in their way of perceiving the world with everything this implies. Spanish classes are essential in the process of shaping the character of a young person as a social person. The development of thinking and cultural awareness can be achieved through the use of an appropriate methodology, in addition to addressing all fields of a foreign language: pronunciation, literature, speaking, listening, reading, vocabulary, written comprehension and a variety of means.
\end{abstract}

Keywords: learning, communication, motivation, storytelling, poems, overcoming limits, theater.

Rezumat. Scopul cercetării este de a sublinia modul în care literatura contribuie la dezvoltatarea interacțiunii sociale a studenților. Factorii cheie pe care ne-am concentrat acoperă întregul domeniu al studierii unei limbi străine prin literatură. Astfel, metodele pe care le sugerăm pentru aceasta se referă la: motivarea cursanților să-și sporească abilitățile de înțelegere a mesajului textelor citite, folosirea poeziilor autentice în predare, crearea unei piese de teatru în clasă, utilizarea textelor dramatice și a narațiunilor. S-a recunoscut în mod constant că noul context european influențează noua generație de studenți în modul lor de a percepe lumea cu tot ceea ce implică acest lucru. Cursurile de spaniolă sunt esențiale în procesul de modelare a caracterului unui tânăr ca persoană socială. Dezvoltarea gândirii și conștientizarea culturală pot fi realizate prin utilizarea unei metodologii adecvate, pe lângă abordarea tuturor domeniilor unei limbi străine: pronunție, literatură, vorbire, ascultare, citire, vocabular, înțelegere scrisă și o varietate de alte mijloace.

Cuvinte cheie: învățare, comunicare, motivație, povestiri, poezii, depășirea limitelor, teatru. 


\section{Introducción}

La motivación es uno de los factores clave que determina el éxito del estudio de una lengua extranjera proporcionando el principal incentivo para iniciar el aprendizaje y, posteriormente, la determinación de perseverar y sostener el largo y, a menudo, difícil proceso de aprendizaje. Sin una motivación suficiente y constante, incluso a las personas con las mejores habilidades le viene enrevesado lograr metas a largo plazo.

El valor intrínseco del aprendizaje de una lengua extranjera está asociado con el interés de los alumnos y estudiantes y disfrute anticipado de la actividad de aprendizaje de idiomas. La clave tema en la generación de interés es abrir el apetito de los estudiantes, es decir, despertar la curiosidad y la atención de los estudiantes y crear un atractivo imagen del curso. Esta es una tarea de "venta" en la que puede señalar aspectos desafiantes, exóticos o satisfactorios del aprendizaje de una lengua extranjera, conectar el aprendizaje con actividades que los estudiantes ya encuentran interesantes o estiman (por ejemplo, aprendizaje asistido por la computadora o juegos electrónicos adaptados para estudiar lenguajes específicos en ciertas situaciones), destacar la variedad de actividades que puede implicar el aprendizaje de una lengua extranjera y proporcione una demostración de algunas tareas particularmente agradables.

Dorney [1] habla de la importancia de hacer que el aprendizaje sea estimulante y agradable. "Las personas suelen estar bastante dispuestas a pasar mucho tiempo pensando y aprendiendo mientras realizan actividades que disfrutan. Solo piense en todas las horas que dedicamos a, digamos, hacer crucigramas, ensayar para representaciones teatrales de aficionados o jugar con la computadora. Estos ejemplos sugieren que el aprendizaje no tiene por qué ser necesariamente una tarea aburrida y tediosa (lo que suele ser). Si de alguna manera pudiéramos hacer que el proceso de aprendizaje fuera más estimulante y agradable, eso contribuiría enormemente a la participación sostenida del estudiante. Esta es una suposición que la mayoría de los psicólogos motivacionales suscriben y que también tiene mucho sentido para los profesores de aula; de hecho, muchos profesionales simplemente equipararían el adjetivo "motivador" con "interesante" (p. 72).

Se supone que los profesores que trabajan en las escuelas o universidades públicas deben enseñar el plan de estudios, pero no podemos ignorar el hecho de que esto no puede suceder sin motivar a nuestros estudiantes. Además, los estudiantes adolescentes y los jóvenes vienen con su propio equipaje e intereses emocionales y psicológicos, por lo que la tarea de motivarlos es uno de los mayores desafíos para los docentes. Utilizar literatura auténtica para complementar los materiales básicos es una forma de motivar a los adolescentes, pero la tarea de leer un cuento o una novela en un idioma extranjero puede resultar abrumadora para muchos estudiantes.

Para garantizar la condición motivacional básica, los profesores deben tener en cuenta tres aspectos principales. Primero, deben crear un ambiente de aprendizaje agradable y de apoyo. Un contexto propicio animará a los alumnos a desarrollar todo su potencial. Luego, deben establecer un código de conducta y normas con las cuales todos estén de acuerdo. Por último, pero no menos importante, deben fomentar los grupos de apoyo entre pares que reconozcan los intereses, niveles, habilidades y fortalezas de cada estudiante. También se debe prestar atención a los resultados finales.

Mantener y proteger la motivación es el siguiente paso que los profesores deben considerar después de aumentar la motivación. En primer lugar, la motivación se puede mantener durante el proceso de lectura ofreciendo tareas durante la lectura con objetivos 
claros, que deben ser lo más variados posible y permitir diferentes formas de respuesta. Esto puede ayudarlos a superar el problema de las clases de niveles e intereses mixtos, involucrar a todos los estudiantes y, por lo tanto, mejorar su autoestima y confianza. En segundo lugar, la lectura debe controlarse. En el primer caso, esto implica comprobar que se ha leído una cierta cantidad de lectura y que se ha entendido lo que se ha leído, y aprovechar esa comprensión para motivar la lectura adicional. Hay que estimular a los estudiantes para que sean conscientes de la importancia de sus predicciones y expectativas en el proceso de lectura. Las opiniones y las interpretaciones deben variar, y su intercambio y evaluación es una parte vital del proceso de aprendizaje interactivo, que involucra el desarrollo del lenguaje, la conciencia cultural y el crecimiento del alumno en términos educativos generales. En tercer lugar, sería bueno que los alumnos llevaran un diario de lectura. Esto tiene la ventaja de ser un registro individual y personal, mientras que al mismo tiempo documenta y refleja el trabajo realizado dentro o fuera de clase. Puede escribirse en el propio idioma de los alumnos, así como parcialmente en español, pero a medida que mejore el nivel de idioma de los estudiantes, se les debería animar a utilizar cada vez más español en sus diarios de lectura. En cuarto lugar, debería integrarse la multimedia. Hay muchas películas y grabaciones de audio que se pueden usar en combinación con una historia. Además, el internet se puede utilizar para investigar autores, entornos y ubicaciones, detalles históricos o información relacionada con el tema.

El uso de la poesía para desarrollar habilidades productivas debe ser tratado con gran seriedad en lo que respecta a los textos auténticos. Los poemas son, después de todo, textos auténticos. Este es un gran motivador. Los poemas son a menudo ricos en referencias culturales y presentan una amplia gama de oportunidades de aprendizaje. Hay dos opciones para los profesores: una es enseñar una lengua extranjera a través de la poesía y la otra es enseñar la poesía en sí. Los profesores deben elegir lo que mejor se adapte a ellos y a sus estudiantes, teniendo en cuenta el concepto de nuevos discípulos en un nuevo contexto europeo. Más adelante nos ocupamos de algunas actividades de expresión y comprensión oral y escrita.

\section{Comprensión oral}

Se podría usar algo de música de fondo para crear la atmósfera, el profesor muestra algunas imágenes para presentar el tema y luego hacer que los estudiantes piensen en su conocimiento o experiencia personal que se relaciona con este tema. Luego hablan sobre el poema, primero con un compañero y luego en grupos pequeños, quizás reuniéndose como clase al final para compartir ideas. El profesor actúa como monitor y aporta ideas y vocabulario si es necesario, da una breve retroalimentación sobre el lenguaje utilizado y toma nota de cualquier problema de lenguaje que se resolverá en una fecha posterior. Las hojas de trabajo para las actividades orales previas a la lectura pueden incluir un cuestionario, frases para completar y discutir, declaraciones para clasificar y discutir, etc. Los estudiantes pueden predecir el final de los versos, el poema completo o eventos que ocurren después del final del poema. Posteriormente, los estudiantes podrían hablar sobre su respuesta personal al poema, discutir los personajes y el tema, o debatir las cuestiones morales. Los juegos de roles funcionan bien, entrevistar a un compañero o incluso dramatizar el poema y hacer un video. Los estudiantes podrían comparar poemas sobre temas relacionados, con diferentes grupos trabajando en diferentes poemas y luego reagrupando sus ideas. 


\section{Comprensión escrita}

Un poema puede conducir a una escritura creativa maravillosa. Los estudiantes pueden agregar más líneas o estrofas individualmente o en parejas o grupos. Pueden escribir una carta a un personaje del poema, escribir sobre lo que sucedió antes del comienzo o después del final del poema, etc. Los estudiantes pueden usar el poema como punto de partida y modelo para una escritura paralela: cada grupo puede contribuir con un verso a un poema colectivo. La transferencia de género presenta muchas oportunidades para la práctica de la escritura; cartas, anotaciones en el diario, obras de teatro de radio, artículos de periódicos, todo basado en el texto original de un poema. Los ejercicios de reformulación pueden resultar muy estimulantes, en los que se cambia entre el lenguaje formal y el informal. Los poemas más largos se pueden resumir en cincuenta palabras. También es divertido hacer que los estudiantes transformen palabras de contenido en sinónimos o antónimos y luego discutan las sutilezas del vocabulario.

Los juegos ayudan al profesor a crear contextos en los que el lenguaje es útil y significativo. Aunque los juegos a menudo se asocian con la diversión, no debemos perder de vista su valor pedagógico, especialmente en la enseñanza y el aprendizaje de lenguas extranjeras. Los juegos son efectivos ya que crean motivación, reducen el estrés de los estudiantes y brindan a los estudiantes de idiomas la oportunidad de una comunicación real. J. Hadfield [2] afirma: "La inclusión de juegos como parte integral de cualquier programa de estudios de idiomas brinda una oportunidad para la práctica intensiva del idioma, ofrece un contexto en el que el lenguaje se usa de manera significativa y como un medio para un fin, y actúa como una herramienta de diagnóstico para el profesor, destacando áreas de interés. dificultad. Por último, pero ciertamente no menos importante, [...] una de las razones más importantes para usar juegos es simplemente que son inmensamente agradables tanto para el profesor como para el estudiante." (p. 5)

Hardfield [2] propone dos formas de clasificar los juegos de lenguaje. Primero, los juegos de lenguaje se dividen en dos tipos: juegos lingüísticos y comunicativos. Los juegos lingüísticos se centran en la precisión, como proporcionar el antónimo correcto. Los juegos comunicativos presuponen un intercambio exitoso de información e ideas. J. Hadfield también ofrece clasificar los juegos de lenguaje en muchas más categorías: ordenar, ordenar u organizar, juegos de brecha de información, adivinanzas, juegos de búsqueda, juegos de emparejar, etiquetar, intercambiar juegos; tablero, juegos de rol. El trabajo en pareja o en grupo es una de las principales formas de incrementar la cooperación.

Muchos juegos se pueden jugar en parejas o en grupos pequeños, lo que brinda la oportunidad de desarrollar sus habilidades interpersonales, como la habilidad de estar en desacuerdo cortésmente o la habilidad de pedir ayuda. En el aula, los estudiantes definitivamente participarán en las actividades. Por lo tanto, en grupos o en parejas, están más dispuestos a hacer preguntas, comunicarse y discutir temas con sus socios y pensar de manera creativa sobre cómo usar un idioma extranjero para lograr sus objetivos. La competencia en los juegos brinda a los estudiantes una oportunidad natural para trabajar juntos y comunicarse mucho entre ellos. Gracias a la motivación y la interacción que generan los juegos, los estudiantes pueden adquirir conocimientos de forma más rápida y eficaz que por otros medios. Los juegos pueden estimular y animar a los estudiantes a participar en la actividad, ya que naturalmente quieren ganar. Además de divertirse, los estudiantes están aprendiendo. Adquieren un nuevo idioma y comienzan a darse cuenta de que tienen que usar el idioma si quieren que otros entiendan lo que están diciendo. Además, los juegos pueden 
reducir la ansiedad. En la atmósfera fácil y relajada que se crea mediante el uso de juegos, los estudiantes recuerdan las cosas más rápido y mejor. El significado del idioma que los estudiantes escuchan, leen, hablan y escriben se experimentará más vívidamente en un juego y, por lo tanto, recordarán mejor el idioma que aprenden.

Crear una obra de teatro en clase y usarla para ayudar a motivar a los estudiantes a desarrollar su pensamiento y aumentar la conciencia cultural es de vital importancia para cualquier profesor de idiomas. Estudiar un idioma extranjero en la escuela puede ser una experiencia completamente deprimente para los estudiantes debido a la traducción de la gramática, los profesores autoritarios y las pruebas constantes. Es posible que recuerden muy poco de lo que se les enseñó, pero algo que nunca olvidarán es su participación en una obra de teatro, cuyas líneas tienen muchas posibilidades de recordar años después. La creación de una obra de teatro en clase debería convertirse en una característica habitual de nuestra enseñanza. Lo primero con lo que debe comenzar es una fecha límite para que todo esté listo. Tal vez podría ser una actuación pública para los padres, el resto de la escuela o un intercambio de clases. Si los profesores tienen un grupo particularmente tímido, pueden restringirlo a unos pocos miembros del personal que vengan a verlo. La fecha límite lo motivará tanto al profesor como a sus estudiantes, y el conocimiento que desempeñarán garantizará que dediquen tiempo a aprender sus líneas. Es aconsejable basar la obra en un tema tratado o en algo que sea de interés actual para los estudiantes. La participación de los estudiantes dependerá de su imaginación y nivel de español. Algunas fotografías de personas y la invención de perfiles pueden ser una buena opción. Los dibujos se pegan en la pizarra y el profesor escribe todo lo que los alumnos le dicen sobre los personajes. Luego discuten juntos las relaciones entre los personajes y una trama básica. Los estudiantes pueden escribir el esquema básico de la obra. A los estudiantes les resulta muy motivador realizar sus propias creaciones. Si los profesores no tienen tiempo o no se sienten cómodos escribiendo una obra de teatro, se puede adaptar una historia familiar que todos sepan. Es fundamental saber elegir el rol más adecuado para cada estudiante. Después de decidir los roles, es importante asegurarse de que todos aprendan sus líneas. La forma más eficaz de hacerlo es practicar cada lección durante diez minutos al principio y al final. Pueden actuar para otras clases, para visitantes y para sus padres. Esto significa que tenían un incentivo real para producir el idioma con precisión y los padres siempre aprecian poder ver a sus hijos hablando en español. Si los estudiantes se sienten cómodos con él, los profesores podrían incluso grabarlo en video o tomar fotografías para usarlas nuevamente en clase después.

Hay varias razones para usar obras de teatro. Son muy memorables, los maestros descubrirán que al final, los estudiantes no solo han aprendido sus partes, sino que también podrán decir la parte de los demás. Los estudiantes aprenderán fragmentos completos de lenguaje en un contexto claro. Es extremadamente satisfactorio para los estudiantes y los padres ver cuánto lenguaje pueden producir. Las obras de teatro brindan a los estudiantes la oportunidad de demostrar otras habilidades en el aula de idiomas. No siempre son los estudiantes más fuertes los que obtienen mejores resultados. Aunque puede ser un poco estresante, puede convertirse fácilmente en una gran experiencia de unión para las clases.

En general, todo el proceso, desde la escritura, la práctica y la interpretación, en realidad no toma tanto tiempo y las recompensas hacen que valga la pena. Algunos estudiantes no querrán una parte hablada, por lo que los maestros deben asegurarse de tener roles adicionales para que los estudiantes tengan alguna opinión. Los profesores y 
estudiantes pueden incluso dedicar tiempo a hacer accesorios y disfraces. Con estudiantes de nivel superior, el proceso de planificación también se puede realizar en inglés.

El uso de textos dramáticos en el aula puede aportar beneficios al aula de aprendizaje de idiomas y puede aprovechar al máximo el potencial de los estudiantes. McRae [3] afirma que actuar y observar dan sustancia al hablar, ya que desarrollan la conciencia de los estudiantes sobre "speech features, paralinguistic and extra linguistic features-attitudes, gestures, intonation, movement, etc." ("características del habla, características paralingüísticas y extralingüísticas: actitudes, gestos, entonación, movimiento, etc."). Discutir, debatir, sugerir interpretaciones, ofrecer opiniones y estar de acuerdo y en desacuerdo "begin to come naturally as the students become more used to using drama and more confident in their use of English" (p. 7). ("comienza a surgir de forma natural a medida que los estudiantes se acostumbran más al teatro y se sienten más confiados en el uso del inglés"). De hecho, todas las actividades inspiradas en obras literarias contribuyen en gran medida a mejorar las habilidades para hablar y escuchar de los estudiantes.

Hay muchas contribuciones positivas al aprendizaje de idiomas a través de la literatura mediante el uso de valioso material auténtico, ya que expone al alumno a diferentes registros y tipos de uso del idioma. El teatro puede ayudar al profesor a alcanzar la realidad de varias formas. Puede superar la resistencia de los estudiantes a aprender el nuevo idioma: haciendo que el aprendizaje del nuevo idioma sea una experiencia agradable, estableciendo objetivos realistas para los estudiantes, vinculando la experiencia de aprendizaje del idioma con la propia experiencia de vida del estudiante. Y el teatro puede crear en los estudiantes la necesidad de aprender el idioma mediante el uso de tensión creativa (situaciones que requieren soluciones urgentes); poniendo más responsabilidad en el estudiante que en el profesor.

El teatro proporciona conciencia cultural y lingüística al revelar conocimientos sobre la cultura de destino y presentar contextos lingüísticos que hacen que los elementos sean memorables colocándolos en un contexto social y físico realista. Uno de los inconvenientes del uso de textos literarios como novelas y poemas es que muchos de ellos contienen formas lingüísticas que los estudiantes de un idioma encuentran difíciles de entender. Esto podría superarse simplificándolos, lo que a menudo conduce a una pérdida de "literariedad", lo que lleva a la crítica de que los textos se convirtieron en pálidas imitaciones del escrito original. Los textos dramáticos ayudan a abordar la necesidad de textos suficientes para una lectura valiosa en los que se pueda acceder a los materiales adecuados.

Usar teatro con estudiantes de idiomas puede mejorar sus habilidades de lectura y expresión oral, fomentar la creatividad, ayudarlos a experimentar con el lenguaje: el tono de voz, el lenguaje corporal y sus propias líneas si participan en la escritura de la obra. A algunos estudiantes les gusta actuar o encuentran que el guion les da confianza. También es una idea involucrar a toda la clase: se pueden dar partes no habladas a los alumnos que no desean hablar o tienen menos confianza. Las partes técnicas de una producción pueden darles un papel a otros: efectos de sonido, hacer escenografía, estar a cargo de las luces, la utilería o incitar a sus compañeros desde las alas.

Los estudiantes pueden crear su propia obra de teatro a partir de una foto, una caricatura o un videoclip, o idear minijuegos basados en personajes reconocibles de películas que les gustan a los estudiantes. Tales ejemplos pueden ser tomar una secuencia de diez minutos de una película, contarles a los estudiantes la historia de fondo, mostrar el clip sin sonido, enfocarse en un par de intercambios y pedirles a los estudiantes que imaginen lo que los 
personajes podrían estar diciendo, asignando roles a parejas. y actuar. Luego, se puede alentar a los estudiantes a que inventen sus propios diálogos breves basados en los roles y que reúnan sus sugerencias y trabajen en un guion de clase.

Por un lado, el análisis del lenguaje en un texto es solo un aspecto de su uso. El lenguaje se separa en sus componentes fonológico, léxico, etc. y luego se adoptan las estrategias de difusión. Con este enfoque analítico, un profesor o un diseñador de cursos pensaría en una serie de estructuras, elementos del lenguaje que debían "enseñarse". A continuación, se selecciona un texto literario que ejemplifica estas estructuras y se utiliza para practicarlo o aumentar la conciencia del alumno al respecto.

Sin embargo, el lenguaje no se ve como un objeto sino como una herramienta. Se pone mucho más énfasis en, por ejemplo, el método inductivo de aprendizaje a través de experimentar y aplicar la experiencia del alumno al texto mediante comentarios alentadores, respuestas y expresiones basadas en el texto o su tema. El programa de estudios basado en un tema a menudo se considera más relevante para el aprendizaje de idiomas.

Maley y Duff [4] enfatizan la importancia de las actividades verbales y no verbales que toman poco tiempo y "están destinadas a hacer que los estudiantes estén de humor para actividades dramáticas más extensas. Más específicamente:

Implican cierto grado de actividad física, lo que ayuda a restablecer el equilibrio entre pensar y hacer.

Ayudan a poner a los estudiantes en un estado relajado, menos inhibido, en el que son más receptivos de lo que podrían ser de otra manera. Esto ayuda a reducir el umbral de resistencia inconsciente al aprendizaje de una lengua extranjera y a fomentar un trabajo más abierto y creativo en las actividades posteriores.

Ayudan a desarrollar la confianza y la cooperación con los demás. Ser consciente de los demás y de cómo nos relacionamos con ellos es un aspecto importante del vínculo de clase.

Pueden ayudar a los estudiantes a realizar una transición sin problemas de sus actividades fuera de la clase (tal vez una lección sobre un tema diferente o el estrés de venir de un trabajo con mucho tráfico) al ambiente de aprendizaje de la clase de idiomas.

También se pueden utilizar para establecer vínculos fluidos entre una actividad y la siguiente.

Hay cuatro tipos principales de actividad:

1 calentamiento no verbal

2 relajación no verbal

3 actividades que involucran el lenguaje

4 actividades de formación grupal.

La forma en que elija utilizar las actividades depende de usted. A medida que se familiarice con el grupo de su clase, sabrá mejor cuáles usar en qué momentos. No hay calificación, aunque las actividades se presentan en grupos cuando comparten elementos comunes ".)

Desde el punto de vista de la tarea, el estudiante se enfrenta a varios niveles de logro o capacidad que el profesor puede utilizar como base para diseñar actividades de varios niveles para los estudiantes:

- Identificar la historia, los personajes, la trama (alcanzable a nivel principiante)

- Identificar el punto de vista, actitud u opinión del autor y de los personajes

- Comprender la obra en relación con su contexto sociocultural e histórico-político 
- Dar una respuesta personal o creativa (por ejemplo, representar el texto)

- Respondiendo a la pregunta: "¿Funciona como literatura?" (Análisis crítico extendido del texto)

La narración tiene muchos beneficios y es muy útil para lograr habilidades de interpretación. Los estudiantes tienen un amor innato por las historias. Las historias crean magia y una sensación de asombro ante el mundo. Las historias nos enseñan sobre la vida, sobre nosotros mismos y sobre los demás. Contar historias es una forma única para que los estudiantes desarrollen comprensión, respeto y aprecio por otras culturas y puede promover una actitud positiva hacia personas de diferentes tierras, razas y religiones. Para Austin [5], para distinguir los usos normales del lenguaje de los usos parasitarios, y de manera similar, para distinguir los usos exitosos de los fallidos, uno debe considerar su contexto total específico, es decir contexto que incluye características internas, como intenciones, y características externas, como una determinada situación social. Los usos del lenguaje no pueden ser infelices o parasitarios independientemente de su contexto total. Estos enunciados tendrán contextos que les sean propios. El contexto total apropiado de un uso del lenguaje es la situación social en la que se usa, pero también debe incluir las intenciones del usuario, ya que algunas expresiones pueden no ser determinables como normales o parasitarias sin tener en cuenta también la intención del emisor.

Hay varias formas en las que la narración puede mejorar la comprensión y la comunicación intercultural. A través de las historias, los estudiantes pueden explorar sus propias raíces culturales, experimentar diversas culturas, empatizar con personas, lugares y situaciones desconocidas. También pueden ofrecer conocimientos sobre diferentes tradiciones y valores, ayudar a los niños a comprender cómo la sabiduría es común a todos los pueblos y todas las culturas, ofrecer conocimientos sobre experiencias de vida universales, ayudar a los alumnos a considerar nuevas ideas y revelar diferencias y similitudes de culturas en todo el mundo. El uso de los libros ilustrados de literatura global con estudiantes en el contexto de un plan de estudios internacional es importante para desarrollar la comprensión intercultural de los jóvenes. Los libros ilustrados, pero también las novelas que se pueden compartir a través de lecturas en voz alta, lectura individual y en pareja y navegación, se centran particularmente en las identidades personales de los estudiantes como seres culturales y sus problemas de identificación por los que toman medidas para hacer del mundo un lugar mejor. Además, se discuten las ilustraciones para desarrollar la comprensión de los estudiantes sobre la creación de significado a través del arte [6]. A través de las experiencias con la literatura global, los jóvenes desarrollan una comprensión más rica de sí mismos como seres culturales complejos y una percepción de sí mismos como capaces de resolver problemas que identifican problemas y toman medidas para resolverlos. El uso de historias en clase tiene muchas ventajas, ya que promueven una sensación de bienestar y relajación, aumentan la disposición de los niños para comunicar pensamientos y sentimientos, fomentan la participación activa, aumentan la competencia verbal, fomentan el uso de la imaginación y la creatividad, fomentan la cooperación entre los estudiantes y mejorar las habilidades de escucha. Las historias revelan verdades universales sobre el mundo. A través de historias, vemos cómo personas muy diferentes comparten las mismas experiencias de vida y cómo la naturaleza humana puede trascender la cultura. Los nuevos estudiantes comparten una notable variedad de experiencias personales, valores y formas de comprensión. El idioma que aprenden en el aula es la herramienta que utilizan para moldear sus pensamientos y sentimientos. Es más que una forma de intercambiar 
información y extender ideas, es su medio de acercarse y conectarse con otras personas. Las historias pueden vincularse no solo entre el mundo del aula y el hogar, sino también entre el aula y más allá. Las historias proporcionan un hilo conductor que puede ayudar a unir culturas y proporcionar un puente a través de la brecha cultural.

Implementar un enfoque basado en historias requiere mucha energía, creatividad y excelentes habilidades de gestión del aula y flexibilidad por parte de los profesores. Al final, los estudiantes presentan su trabajo a los padres o talvez a sus amigos y compañeros, lo que proporciona una forma ideal de fortalecer las relaciones entre ellos. Sin embargo, esto puede poner a los profesores bajo cierta presión, ya que el desempeño de sus estudiantes a menudo se equipará con su desempeño como profesores. En otras palabras, mantiene una enseñanza de idiomas de alta calidad. Inicialmente, a algunos profesores les resulta difícil imaginar cómo pueden usar un libro durante un máximo de 6 a 10 horas. Sin embargo, una vez que han utilizado la literatura, a menudo les resulta difícil volver a los materiales de español como lengua extranjera más convencionales y específicamente escritos, que tienden a permanecer en el nivel mundano y utilitario de los diálogos básicos y las actividades diarias. Las novelas y los libros de cuentos abordan temas universales y permiten a los estudiantes jugar con ideas y sentimientos y pensar en temas importantes. Para concluir, ver la literatura como un proceso y considerar cómo se interrelacionan cada una de las diferentes etapas puede ayudar a nuestros estudiantes a desarrollar el hábito de la lectura al volverse más conscientes de sí mismos, positivos, competentes y autónomos y, en consecuencia, motivados para abordar el próximo texto auténtico.

\section{Bibliography}

1. Dornyei Z. Motivational Strategies in the Language Classroom. Cambridge: Cambridge University Press, 2001.

2. Hadfield J. Beginners' communication games. Longman, 2010.

3. Mcrae J. Using Drama in the Classroom. Pergamon Press, 1985.

4. Maley A., Duff A. Drama Techniques. A Resource Book of Communication Activities for Language Teachers, Cambridge: Cambridge University Press, 2010, available at Getting ready (Chapter 1) - Drama Techniques (cambridge.org)

5. Austin J.L. How To Do Things With Words. Harvard University Press, 1962.

6. Martens P. Building International Understanding through Global Literature [online] 2015, available at Building Intercultural Understandings Through Global Literature - Martens - 2015 - The Reading Teacher - Wiley Online Library

7. Madrid C. Misterio En El Teatro - Mystery in The Theater (Spanish- English Edition): Learn Spanish Easily By Reading. Beginners A1-A2 (Pepa Torres, periodista (Spanish- English Edition)) (Spanish Edition), Independently published, 2019

8. Eisenmann Maria. Teaching English: Differentiation and Individualisation, Brill Group, 2019,

9. Thornbury S. How to Teach Vocabulary. Harlow: Longman, 2002

10. Seedhouse P. \& Jenks C.J., International Perspectives on ELT Classroom Interaction: An Introduction. Houndmills, Basingstoke, Hampshire: Palgrave Macmillan, 2015

11. Porto M., Byram M. New Perspectives on Intercultural Language Research and Teaching: Exploring Learners' Understandings of Texts from Other Cultures. Routledge, 2016

12. Trujillo F. (ed). Aprender y enseñar en tiempos de confinamiento. Los libros de la Catarata, 2020

13. Blake J.R, Zyzik E.C. El español y la lingüística aplicada. Georgetown University Press, 2016

14. Paredez Garcia F., Alvaro Garcia, S., Paredez Zurdu L. Las 100 dudas más frecuentes del español. Instituto Cervantes, Edelsa, 2019

15. Castillo J.R. Textos literarios y enseñanza del español. Universidad Nacional de Educación a distancia, 2013 